\title{
How Do Writing Tools Shape Interactive Stories?
}

\author{
Sofia Kitromili, James Jordan and David E. Millard \\ University of Southampton, Southampton, UK \\ (sk1n15, dem, jaj1)@ soton.ac.uk
}

\begin{abstract}
Interactive Digital Storytelling is a diverse field, with a variety of different tools and platforms, many of them bespoke. Understanding how these tools effect the stories created using them would allow authors to better select tools for projects, and help developers understand the consequences of their design decisions. We present an initial exploration of this question, using a critical reflection method to analyze the process of adapting a story from StoryPlaces into both Twine and Inform 7 . We report four significant differences that posed challenges for adaptation: support for rewinding and/or revisiting, the definition and description of locations, the way in which text is delivered to the reader, and how navigational cues are provided to help readers progress the story. Our observations show that tools impact the stories created using them in ways that are not obvious when working with one platform alone.
\end{abstract}

Keywords: Interactive Storytelling, Writing Tools, Interactive Story Authoring, Hypertext Fiction, Interactive Fiction, Location Aware Narratives.

\section{Introduction}

Digital interactive writing tools are systems used to author digital stories. The stories produced are interactive and typically non-linear. While there are a number of established systems, digital interactive storytelling is an experimental form, with a wide variety of different platforms and writing tools available. This raises an interesting question: how does the design of writing tools impact the types of stories being written? Researchers have studied the reading experiences and commented on improvements for interactive writing tools based on that experience [1][2], but the authoring experience, and how it is mediated by tools, remains relatively unexplored [3].

This paper describes initial work to understand how different interactive writing platforms impact the authoring process, and ultimately shape the work of writers using those platforms. To approach this, we experimented with adapting an interactive story from one platform into two very different platforms. Our goal was to reflect on the way in which different tool affordances impacted the way in which the story was told. As such our methodology is one of critical reflection research [4] a reflexive qualitative approach that is strongly related to action research [4], and which produces insights into the researcher's own experience. The story we chose to adapt was Fallen Branches by Katie Lyons [5], a locative story set in Crystal Palace Park, and originally designed and deployed with the StoryPlaces platform [6]. In our work we adapted the story into 
Twine [7] an open source hypertext fiction tool with a graphical authoring interface and no explicit modelling of location, and Inform 7 [8] an interactive fiction system that uses a natural language interface and has a virtual world model (similar to classical MUDs).

\section{Background}

While interactive fiction has its roots in non-digital experimental storytelling, the first digital stories to attract critical interest were those produced on the Storyspace platform [9], of which perhaps the best known is Michael Joyce's afternoon, a story. Storyspace represents the classical node-link model of hypertext, with hotspots appearing in the text that navigate the reader to another node. Storyspace stories can thus be represented as a network of nodes that can be analysed to reveal particular patterns of authorship [10]. The node-link model is only one example of how to create interactive narratives (albeit the dominant one). Bernstein calls the model 'calligraphic' as authors draw explicit navigational paths between nodes, and suggests that an alternative model might be 'sculptural' where all nodes are potentially linked, but rules and conditions sculpt away those links at runtime [10]. Sculptural hypertext systems include StoryPlaces, a locative hypertext system that includes location as one of the condition criteria [6] and StoryNexus by Failbetter Games (the engine behind Fallen London) where the approach is called Quality-Based [11]. Inform 7 also uses a world-model and rules to evaluate reader inputs, but is far more dynamic in nature than these other sculptural hypertext tools, producing an experience which is as game-like as it is hypertextual.

Spatial Hypertext is another alternative approach where text and structure are graphically manipulated into lists, groups and sets [12][13]. Although rarely applied to narrative (with a proper spatial parser), spatial hypertext has influenced modern tools, such as StorySpace 3 and Twine, which embrace a graphical authoring paradigm.

The impact of different data models and tools on authoring is not well explored in the literature, perhaps because it is so difficult to separate the author experience, the requirements of a particular story, and the impact of the tool itself. For example, Hargood et al. attempted to investigate how a novel model called Fractal Hypertext might impact story choices and themes chosen by a set of authors, but concluded that poetics emerge gradually through use and that with novel models this is not mature enough to understand the relationships [14]. We are attempting to explore the same idea, and for this reason have chosen two established tools as target platforms for our work. StoryPlaces, our source platform, is locative and sculptural. While our target platforms are Twine, non-locative and calligraphic; and Inform 7, virtual locations and rules-based.

\section{Methodology}

Fallen Branches was first published on the StoryPlaces platform in 2017. As a short story of around ten thousand words it is of manageable size and complexity for our purposes. 

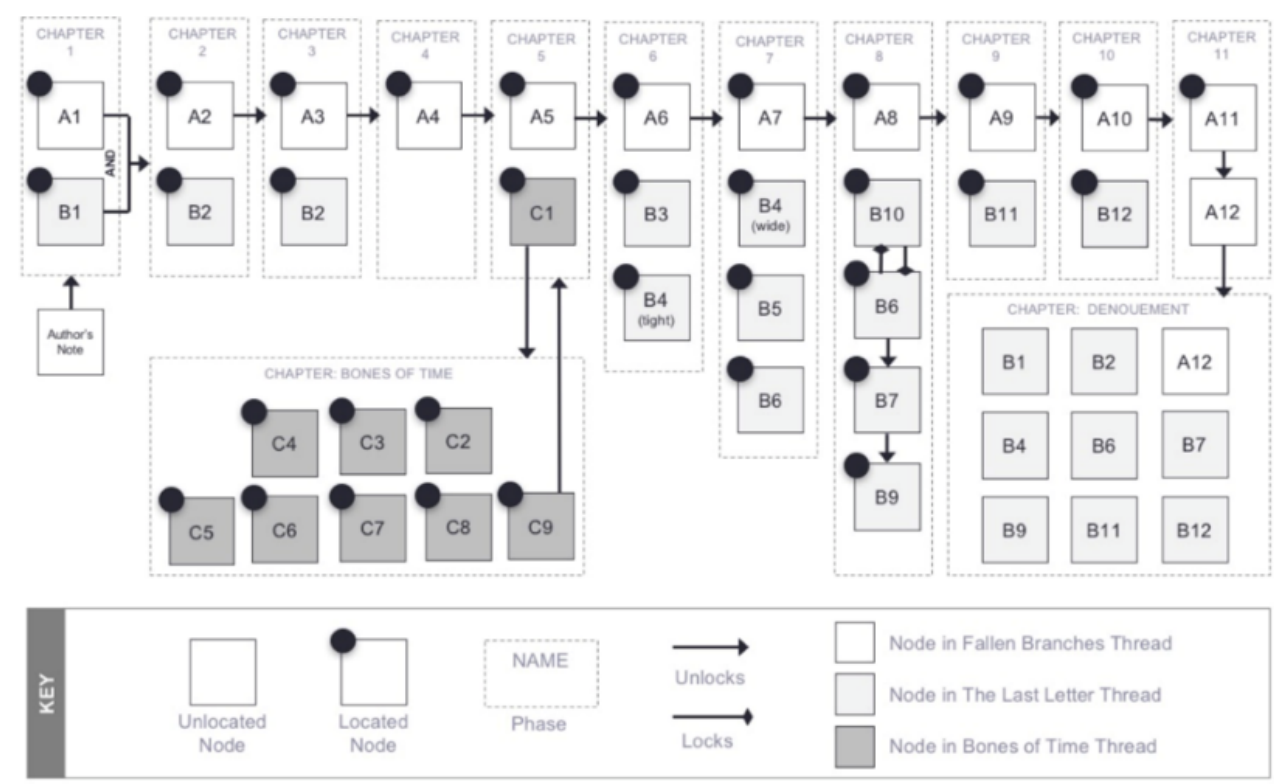

Figure 1 - Fallen Branches sculptural structure (taken from [5])

Figure 1 shows the structure of Fallen Branches. It is composed of 11 chapters with each one containing non-consecutive located nodes. Each is part of one of three story threads; the main Fallen Branches thread, an account of a young woman called Sandy who is visiting Crystal Palace Park to meet with the museum curator and find out more about some family heirlooms; the Last Letter thread, a sequence of letters written between young lovers around the time of World War 1; and the Bones of Time, which recounts an episode in 1900 when two elephants escaped from the Crystal Palace. The reader moves through the park following Sandy, while the letters appear in the landscape around them. The tale of the elephants is effectively a sub-story that is available half-way around, and which temporarily switches the narration back to 1900 .

We obtained the original JSON representation of Fallen Branches, and undertook an exercise to adapt it, firstly for Twine, and then for Inform 7. In both cases a structure model was created similar to the one in Figure 1.

Throughout our adaptation process we met to brainstorm ideas and record our observations and decisions. Figure 2 shows the structure of the story in Twine. Passages in Twine are named after the titles of nodes in each chapter of Storyplaces, and links were created whenever navigation between those nodes was possible in the original story. As Twine contains no explicit representation of locations this does not appear in the Twine map, relying instead on the text to orientate the reader. It is interesting to note the complex relationships between the Bones of Time nodes (right and bottom) which exist because these can be visited in any order. In StoryPlaces sculptural system these are simple to model (there are no requirements so all are available throughout the 
sub-story until they are read) but in Twine's calligraphic system it results in a complex tangle of links [9].

With Inform 7 the process was quite different. In Inform 7 the process is to build a world model, which the reader can then explore (for example, using shortcuts such as ' $N$ ' to go north). The first step was to recreate the locations from Crystal Palace in as a series of rooms, with navigational routes between them that reflected their positions in the real world.

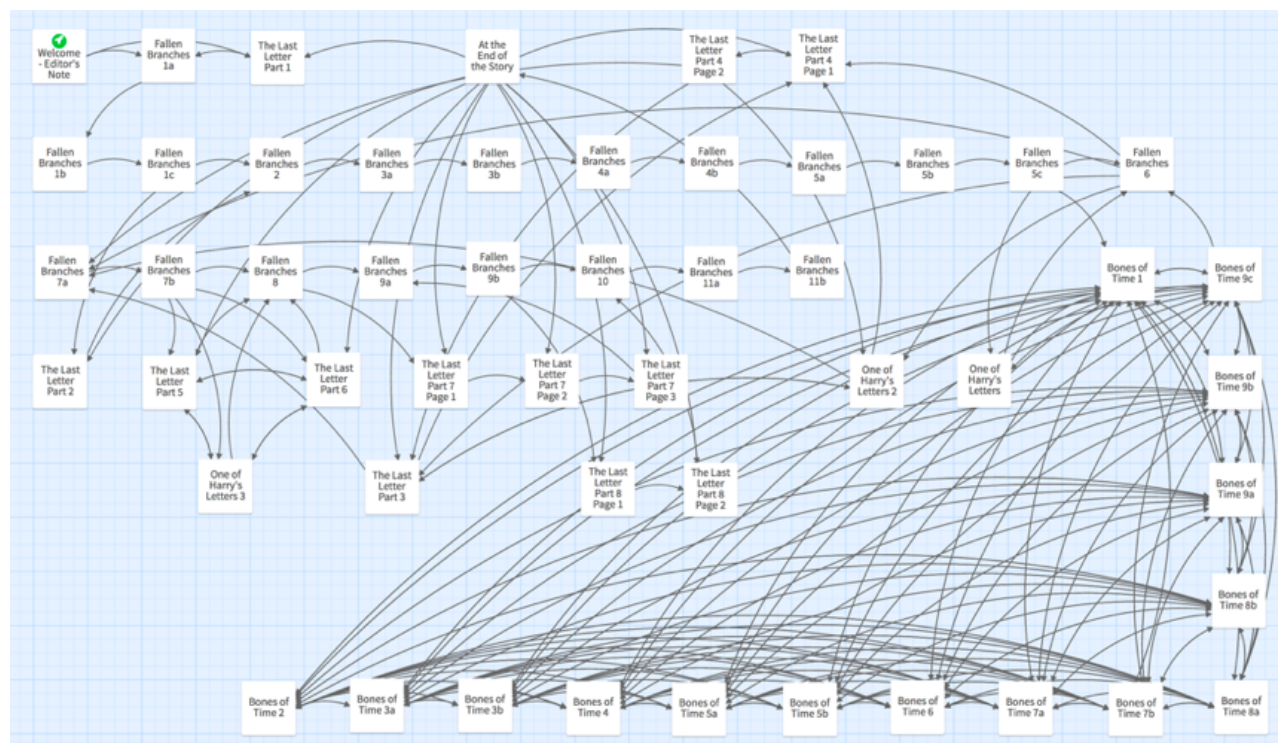

Figure 2 - Fallen Branches structure in Twine

Figure 3 shows the navigational connections between locations authored as rooms in Inform 7. Inform 7 has another construct, Scenes, which are used to represent logical parts of a story that can be sequenced together (for example, acts of a story). In our adaptation, we translated the StoryPlaces chapters into scenes. This means that although the reader is free to move around the locations (as they are in StoryPlaces), the story itself plays out in a more sequential manner as dictated by where the scenes are set. Finally, Inform 7 has the notion of objects that exist within the story world and can be picked up and moved around. We used objects to represent the last letters, these are revealed and added to the users' inventory when first arriving in the correct room in the correct scene.

As described above Fallen Branches contains three story threads. The only path that readers must read to complete the story is the Fallen Branches thread. The rest are dependent on the path that the readers follow. The Last Letters are revealed and unlocked at specific locations as well as the Bones of Time sequence. If a letter is missed and the reader moves on, the letter cannot be discovered at a later point. If the Bones of Time story is activated, readers must complete reading all those events before returning to the main story. The notion of this has been adapted while authoring the story in the 
other writing tools. In Twine all three threads have been implemented behind nodes which point to each other. In Inform 7 scenes where created to resemble chapters, rooms to resemble locations and items to resemble letters.

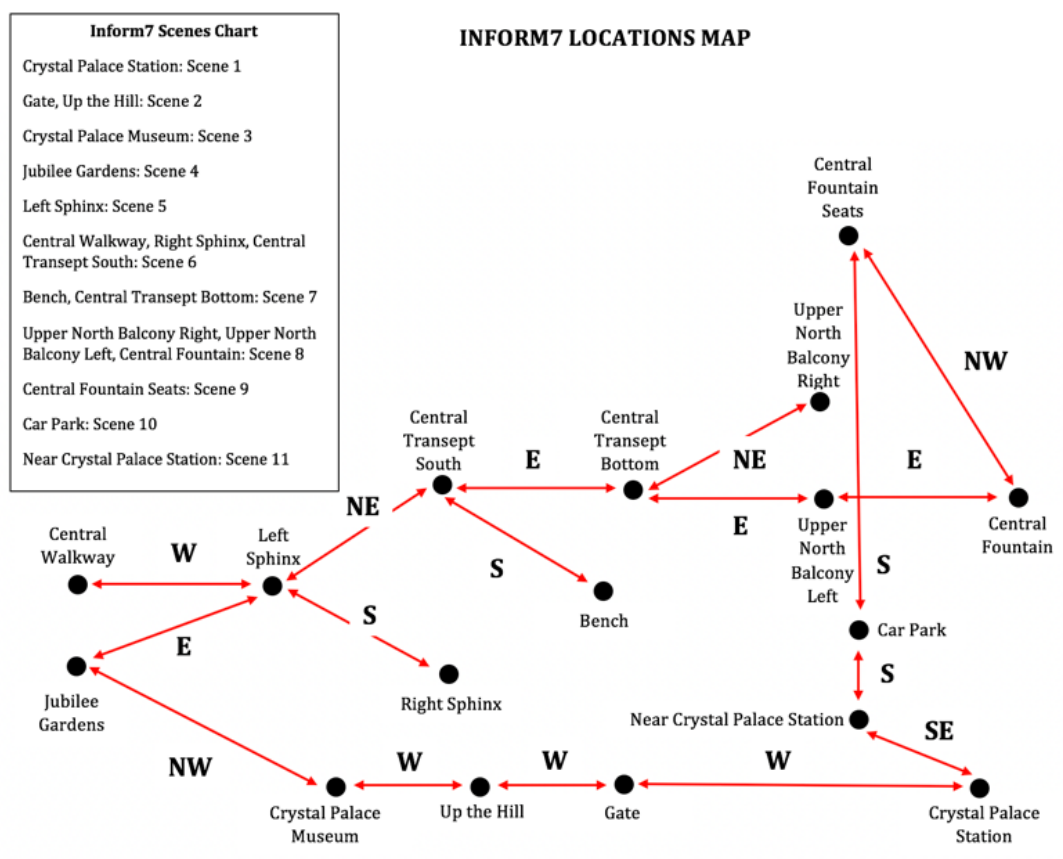

Figure 3 - Fallen Branches navigational structure

\section{Analysis}

During the adaptation process we observed how the tools impacted on the telling of the story. We were interested in cases where narrative design decisions made in StoryPlaces were difficult to replicate in the other models, and where the original author had made assumptions about how the story would be delivered that needed additional work to replicate or replace in the other tools. These points of tension are a good indicator of how the story has been shaped by the tool itself.

Observation 1: Rewinding and Revisiting: In StoryPlaces if the reader failed to read one of the Last Letter nodes then the letter would disappear from the map and the reader wouldn't be able to access it, even if they return to that location. Similarly, if the reader unlocks the first Bones of Time node the rest of the nodes disappear and do not reappear until the reader completes the sequence. We translated this logic to explicit links in Twine. Where a node was made available in Storyplaces a hyperlink was made 
available in Twine and when a node disappeared so did that hyperlink. However, Twine by default includes a back button in all the stories so that the reader can easily rewind to any state in the story and revisit missed nodes. Storyplaces allows these restrictions to give the reader momentum through the landscape, and prevent them doubling back, which is both time consuming and tiresome in real locations [5]. There are two functions here. Revisiting - meaning to return to a previous location and Rewinding - meaning to revert the story back to a previous state. Revisiting locations is possible in StoryPlaces, but rewinding nodes is not. Whereas Twine enables both revisiting and rewinding, and makes only a minor distinction between them (as nodes and locations are tied together). Similar to StoryPlaces, Inform 7 readers can navigate and revisit any location they want, but there is no way to take the narrative back to a previous state (as controlled via scenes), so revisiting is supported, but rewinding is not.

Observation 2: Definition and Description of Locations: All the locations mentioned in Fallen Branches are real places, identified via GPS co-ordinates and communicated to readers through a map interface. As a result, locations need to be defined, but not described, and there is no need to define the connections between them. Neither Twine nor Inform 7 use real world locations and therefore describing locations and connecting them together needs to be done manually. Inform 7 is based on a world model, and this can be done by using rooms and defining how they relate to one another. Twine has no location model at all, therefore locations need to be represented in the text of the nodes themselves (if at all). The text of Fallen Branches makes passing reference to specific locations (for example the museum) and in our judgement meant we did not need to add any additional location information. Should we have added additional text to describe locations and re-create the sense of place? The story clearly loses context as a result of not doing this, but there is no way of knowing what an author intended by placing a node in a location in the first place - or whether that intention is what readers took from the experience. If we made that obvious, either in Twine's text or Inform 7's location descriptions, we would be guessing at something that in a locative work is actually co-created by the reader and the real-world place.

Observation 3: Text Delivery: Text in Storyplaces is represented as pages tied to locations on the map, these are unlocked when a reader visits their location. The text can be of any form, and in Fallen Branches it appears as either a page describing the contemporary visit to the park, a page from an historical account of the elephant escape, or the text of a letter. This translates well into Twine, where individual nodes can also take different forms. However, text in Inform 7 is tied to the world model. For the reader to encounter the text, they must interact in some way with that model. For the visit story, and the elephant escape, we used the room description to convey the text. However, this does not work so well for the letters. To solve this, we added the letters to the world-model as objects. Rather than place them in the rooms to be discovered (which makes no narrative sense, as they are supposed to be in Sandy's possession from the beginning), we created them in particular rooms and automatically add them to Sandy's inventory at the appropriate room and scene in the story. A more radical adaptation of the story for Inform 7 would have taken this further, and taken more of the node content from the other threads and integrated that with the world model (for example, in descriptions of specific objects, or interactions with characters). But in doing so it would 
have fundamentally changed the way in which the story was experienced, and moved the authorial task towards a process akin to hiding treasure. The need to imaginatively integrate the text in this way is a significant difference between Inform 7 and Twine and StoryPlaces, and reflects Inform 7's game-like structure.

Observation 4: Navigational cues: When reading in Storyplaces the system shows locations on a map that when visited would unlock appropriate content to progress the story. The map therefore acts as a navigational cue. In theory readers can move anywhere, but the map shows which places they should visit next. In Twine the locations are tied to the nodes, it's impossible to move around locations without moving between story nodes, and therefore the only navigation that is possible are the links that progress the story. However, in Inform 7 the reader can still navigate around the defined locations, but there are no navigation cues to show which of those possibilities will progress the story. Writing in Storyplaces or Twine means that the author does not need to worry about these cues, whilst writing in Inform 7 means that they are important consideration and must be included in the story world somehow - otherwise how does the reader know what to do next? In our adaptation to solve this problem we used the Inform 7 description of rooms. When a reader visits a room a description of that room is displayed on the screen. That description is the text of the Fallen Branches story as it appears when activated in location. To enable cues, we added a check on the current scene, replacing or supplementing that text with an appropriate cue for the next scene. The presence of automatic cues in StoryPlaces and Twine is a major difference to Inform 7 , where the author is responsible for making progress clear.

\section{Conclusion}

Understanding how tools influence interactive writing is a key challenge in the area of interactive digital storytelling. This potentially influences the choice of tools for particular projects, the design and development of new platforms, and critical analysis of interactive works. Developing an understanding is challenging, as authoring experience is subjective, and different creative projects have different requirements and therefore generate different frictions.

In this paper we have undertaken an initial exploration of the problem through critical reflection research. Our task was to adapt a story from one interactive form to two others, to reflect on that experience, and to use the process to identify differences between tools that might impact the ways in which an author choses to tell a story. We have presented four differences that we observed between the tools in the experiment: support for rewinding and/or revisiting; the way in which locations are defined and described; the way in which text is delivered to the reader, and the way in which navigational cues are provided to the reader that help them to progress the story. Going in to the activity we expected StoryPlaces and Inform 7 to be closer in character, as both emphasize location, but our findings show a more complex picture. It is true that both StoryPlaces and Inform 7 do not allow rewinding, and decouple location from nodes, but in other ways they are more similar to Twine than each other. For example, Twine and Inform 7 are clear in how location is used and impacts story, whilst StoryPlaces is 
more ambiguous - meaning the author has far less control. Twine and StoryPlaces also require less of the author in terms of providing navigational cues, and neither requires the author to consider text delivery, as both have a single mechanism for showing readers content. These last two characteristics could be said to separate the more traditional hypertext approach of StoryPlaces and Twine, from the environmental storytelling [15] aspects of Inform 7.

While this paper represents only initial work, it does demonstrate that differences in the impact of narrative models on the authoring task. These can begin to be identified, and their subsequent effect on the stories themselves explored. By testing a small sample of writing tools, we have discovered observations that work limited to one tool could not provide. One of the most valuable aspects of interactive digital narratives is the diversity of the form itself, represented by the different authoring tools and models in use, and we believe that efforts to develop theory around digital narratives and authorial practice can embrace this diversity and develop it as a strength.

\section{References}

1. J. Pope, "How do readers interact with hypertext fiction? An empirical study of readers' reactions to interactive narratives.", phd, Bournemouth University, Fern Barrow, Poole, Dorset, BH12 5BB, UK, 2007.

2. R. Schneider, "Hypertext narrative and the reader: a view from cognitive theory," Eur. J. Engl.Stud., vol. 9, no. 2, pp. 197-208, Aug. 2005.

3. H. Koenitz, "Three Questions Concerning Authoring Tools," in Proceedings of the 10th International Conference on Interactive Digital Storytelling Workshops, Madeira, Portugal, 2017, p. 4.

4. C. Morley, "Critical Reflection as a Research Methodology," in Knowing Differently: Arts-based and Collaborative Research Methods, 2008, pp. 265-280.

5. H. Packer, C. Hargood, Y. Howard, P. Papadopoulos, and D. Millard, "Developing a Writer's Toolkit for interactive locative storytelling," in Proceedings of the International Conference on Interactive Digital Storytelling, 2017, vol. 10690, pp. 63-74.

6. C. Hargood, M. J. Weal, and D. E. Millard, "The StoryPlaces Platform: Building a Web-Based Locative Hypertext System," in Proceedings of the 29th on Hypertext and Social Media, New York, NY, USA, 2018, pp. 128-135.

7. "Twine / An open-source tool for telling interactive, nonlinear stories." [Online]. Available: http://twinery.org/. [Accessed: 03-May-2018].

8. “Inform." [Online]. Available: http://Inform 7.com/. [Accessed: 03-May-2018].

9. M. Bernstein, "Structural Patterns and Hypertext Rhetoric," ACM Comput Surv, vol. 31, no. 4es, Dec. 1999.

10. M. J. Weal, M. Bernstein, and D. Millard, "On Writing Sculptural Hypertext," 2002.

11. E. Short, "Beyond Branching: Quality-Based, Salience-Based, and Waypoint Narrative Structures," Emily Short's Interactive Storytelling, 12-Apr-2016. . 
12. C. C. Marshall and F. M. Shipman, "Spatial hypertext: designing for change," Commun. ACM, vol. 38, no. 8, pp. 88-97, Aug. 1995.

13. C. Atzenbeck, T. Schedel, M. Tzagarakis, D. Roßner, and L. Mages, "Revisiting Hypertext Infrastructure," in Proceedings of the 28th ACM Conference on Hypertext and Social Media, New York, NY, USA, 2017, pp. 35-44.

14. C. Hargood, R. Davies, D. Millard, M. R. Taylor, and S. Brooker, "Exploring (the poetics of) strange (and fractal) hypertexts," presented at the 23rd ACM Conference on Hypertext and Social Media, 2012.

15. H. Jenkins, "Game Design as Narrative Architecture," Computer, vol. 44, Jan. 2002. 